\title{
Die Verwaltungsanleitung (Xingzheng zhidao) im chinesischen Recht
}

\author{
Von Daniel Krause, Köln*
}

\section{Einleitung}

Die mediale Berichterstattung zu China bezieht sich zumeist auf Politik und Wirtschaft. Wird dem chinesischen Rechtssystem Aufmerksam zuteil, so betrifft dies in der Regel mangelhafte Garantien von Grundrechten oder diverse Aspekte des Wirtschaftsrechts. Das Verwaltungsrecht, das sich in China seit einiger Zeit entwickelt, wird höchstens am Rand behandelt. Dieser Beitrag stellt ein Rechtsinstitut vor, welches sich gewissermaßen zwischen diesen Bereichen bewegt und als ein „Kind beider Welten“ sowohl von wirtschaftsund sozialpolitischem als auch (verwaltungs-)rechtsdogmatischem Interesse ist.

Hinter dem terminus technicus „Verwaltungsanleitung“" (xingzheng zhidao) verbirgt sich das funktionale Äquivalent dessen, was in Deutschland seit rund 30 Jahren unter ,informalem Verwaltungshandeln““2 firmiert. Nachdem sich ein beachtliches Schrifttum ${ }^{3}$ dazu angesammelt hatte, wurde der Fokus auch auf das Ausland ausgedehnt. Schnell fand man im japanischen Recht ein ähnliches Phänomen. Der sogenannten ,administrative guidance“ (japanisch „gyôsei shidô") wurde in der Folgezeit einige Beachtung zuteill ${ }^{4}$, was

Daniel Krause, Diplom-Regionalwissenschaftler (Ostasien) und wissenschaftliche Hilfskraft am Institut für neuere Privatrechtsgeschichte, Deutsche und Rheinische Rechtsgeschichte der Universität zu Köln. E-mail: Daniel.Krause@uni-koeln.de

Kritisch zur Übersetzung des japanischen „gyôsei shidô“ bzw. des chinesischen „xingzheng zhidao“ als „Verwaltunganleitung“ Wolfgang Pape, Gyoseishido und das Anti-Monopol-Gesetz in Japan, Köln 1980, p. 6f; Julia Walkling, Informelles Verwaltungshandeln in Deutschland und Japan. (Re-) Formalisierung des Informellen? Rechtsvergleichende Untersuchung am Beispiel der Verwaltungspraxis bei der Genehmigung von Abfallbeseitigungsanlagen, Frankfurt a.M. 2005, p. $53 f$.

Insoweit wohl begriffsbegründend Eberhard Bohne, Der informale Rechtsstaat - Eine empirische und rechtliche Untersuchung zum Gesetzesvollzug unter besonderer Berücksichtigung des Immissionsschutzes, Berlin 1981.

Vgl. vertiefend Michael Fehling, Informelles Verwaltungshandeln, in: Wolfgang Hoffmann-Riem; Eberhard Schmidt-Aßmann; Andreas Voßkuhle (eds.), Grundlagen des Verwaltungsrechts - Bd. 2: Informationsordnung, Verwaltungsverfahren, Handlungsformen, München 2008, p. 1341ff; zahlreiche Nachweise bei Hartmut Maurer, Allgemeines Verwaltungsrecht, 18. Aufl., München 2011, § 15, Rn. 24; Hans J. Wolff / Otto Bachof / Rolf Stober / Winfried Kluth (eds.), Verwaltungsrecht II - Ein Studienbuch, 7. Aufl., München 2010, § 57, vor Rn.1.

4 Vgl. z.B. Gesine Foljanty-Jost, Informelles Verwaltungshandeln: Schlüssel effizienter Implementation oder Politik ohne Politiker?, in: Ulrich Menzel u.a. (eds.), Im Schatten des Siegers: Japan Band 3 Ökonomie und Politik, Frankfurt a.M. 1989, p. 171ff; Tokiyasu Fujita, Streitvermeidung und Streiterledigung durch informelles Verwaltungshandeln in Japan, Neue Zeitschrift für Ver- 
auch damit zusammenhängen mag, dass das deutsche und das japanische Verwaltungsrecht gewissermaßen zusammen aufgewachsen sind. ${ }^{5}$ Darüber hinaus kam unter den ostasiatischen Staaten Japan in den letzten beiden Dekaden des 20. Jahrhunderts wirtschaftlich auch immense Bedeutung zu. ${ }^{6}$ Angesichts der stetig wachsenden wirtschaftlichen und weltpolitischen Bedeutung Chinas ist es heute gerechtfertigt und geboten, auch das informale Handeln des jungen chinesischen Verwaltungsrechts, näher zu beleuchten. Bis heute ist dies, soweit ersichtlich, im deutsch- und englischsprachigen Bereich nur sehr am Rande geschehen. $^{7}$

\section{Informales Verwaltungshandeln im System des chinesischen Verwaltungsrechts}

\section{Gesetzgebung}

Das chinesische Verwaltungsrecht in seinem traditionellen Sinne hat zwar weit zurückreichende historische Wurzeln, die Entwicklung eines rechtsstaatlichen Verwaltungsrechts befindet sich jedoch noch in der Anfangsphase. Zwar wurden bereits grundlegende Gesetze erlassen, es fehlt jedoch auch nach langjährigen Bemühungen an einem allgemeinen Verwaltungsverfahrensgesetz. ${ }^{8}$ Als Rechtsquellen dienen daher eine Reihe von Einzelgeset-

waltungsrecht (NVwZ) 1994, p. 133ff; Yoichi Ohashi, Verwaltungsvorschriften und informelles Verwaltungshandeln: Zum besseren Verständnis der Verwaltungspraxis Japans, Verwaltungsarchiv (VerwArch) 1999, p. 221ff; Yoishi Ohashi, Gyosei shido und Sanktionen, Zeitschrift für japanisches Recht (ZJapanR) 1999, p. 43ff; Pape, note 1, p. $3 \mathrm{ff}$.

Vgl. z.B. Bin Takada et al., Hauptprobleme des japanischen Verwaltungsrechts, VerwArch 1978, p. $34 \mathrm{ff}$.

6 Vgl. zu den historischen Ursachen Erich Pauer, Die Industrialisierung Japans, in: Peter Feldbauer et. al. (eds.), Industrialisierung - Entwicklungsprozesse in Afrika, Asien und Lateinamerika, Frankfurt a.M. 1995, p. 83-99; zu den Perspektiven Werner Pascha, Perspektiven und Probleme der japanischen Wirtschaft aus langfristiger Sicht, in: Werner Draguhn (ed.), Chinas und Japans Bedeutung für Ostasien und die Weltwirtschaft, Hamburg 2003, p. 79ff.

Sehr kurz lediglich bei Robert Heuser, Einführung in die chinesische Rechtskultur, 2. Aufl., Hamburg 2002, p. 291f; ders. „Sozialistischer Rechtsstaat“ und Verwaltungsrecht in der VR China (1982 - 2002), Hamburg 2003, p. 67f; ders., Verwaltungsrecht, in: Brunhild Staiger / Stefan Friedrich / Hans-Wilm Schütte (eds.), Das große China-Lexikon - Geschichte, Geographie, Gesellschaft, Politik, Wirtschaft, Bildung, Wissenschaft, Kultur, Hamburg 2003, p. 807; Fei Liu, Die gerichtliche Verwaltungskontrolle als Entwicklungsfaktor des chinesischen Verwaltungsrechts - Eine vergleichende Untersuchung zwischen China und Deutschland, Frankfurt a.M. 2003, p. 105.

8 Zum Problemkomplex vgl. Robert Heuser (2003), note 7, p. 68ff; zur Notwendigkeit vgl. Songnian Ying, Die Entwicklung von Bindung der Verwaltung an Recht und Gesetz in China, in: Deutsch-Chinesischer-Rechtsstaatsdialog (ed.), Rechtsbindung der Verwaltung und Individualrechtsschutz - Beiträge zum Eröffnungsseminar des deutsch-chinesischen Rechtsstaatsdialoges vom 12.-13.06.2000 in Peking, p. $65 \mathrm{ff}$. 
zen. ${ }^{9}$ Herausragende Bedeutung kommt unter diesen dem 1990 in Kraft getretenen Verwaltungsprozessgesetz (VPG) ${ }^{10} \mathrm{zu}^{11}{ }^{11}$ Nachdem von 1978 bis dato in der Hauptsache lediglich Regelungen zur internen Verwaltungsorganisation erlassen worden waren, war mit dem VPG zum ersten Mal in der Geschichte der Volksrepublik ein Gesetz in Kraft getreten, das dem Bürger systematische, wenn auch nicht flächendeckende, Rechte und Rechtsschutzmöglichkeiten gegen den Staat garantierte. ${ }^{12}$ Noch in den 1990er Jahren wurden das Staatshaftungsgesetz (1994) ${ }^{13}$, das Verwaltungsstrafgesetz (1996) ${ }^{14}$ und das Verwaltungswiderspruchsgesetz (1999) ${ }^{15}$ erlassen. Im neuen Jahrtausend folgten das Gesetzgebungsgesetz $(2000)^{16}$, Verwaltungsgenehmigungsgesetz $(2003)^{17}$ und, jüngst am 01.01 .2012 in Kraft getreten, das Verwaltungszwangsgesetz $(2011)^{18}$.

\section{Handlungsformen}

Der Verwaltungsakt ist die dominierende und zentrale Handlungsform der chinesischen Verwaltung. ${ }^{19}$ In der chinesischen Verwaltungsrechtsdogmatik werden konkrete (juti) und abstrakte (chouxiang) Verwaltungsakte (xingzheng xingwei) unterschieden. Die Abgrenzung kann mangels Legaldefinitionen zwar nicht zweifelsfrei geleistet werden, nähere

Vgl. Yuanshi Bu, Einführung in das Recht Chinas, München 2009, p 44f.

Zhonghua remin gongheguo xingzheng susongfa (1989), Guowuyuan gongbao (Ggb) [Amtsblatt des Staatsrates] 1989, p. 297ff; deutsche Übersetzung bei Heuser (2003), note 7, p. 244ff.

Vgl. Robert Heuser, „Sozialistisches Recht“ in der Erprobung, Zeitschrift für chinesisches Recht (ZChinR) 2009, p. 256f; Randall Peerenboom, Globalization, Path Dependency and the Limits of Law: Administrative Law Reform and Rule of Law in the People's Republic of China, Berkeley Journal of International Law (BJIL) 2001, p. 224.

Vgl. Jiefen Li, Legal Reform versus the Power of the Party and State in the People's Republic of China. Rule of Law or Rule by Law?, New York 2008, p. 57f.

Zhonghua renmin gongheguo guojia peichangfa (1994), Zhongguo falü nianjian (ZGFLNJ) [Juristisches Jahrbuch der Volksrepublik China] 1995, p. 163ff; deutsche Übersetzung bei Heuser (2003), note 7, p. 299ff.

Zhonghua renmin gongheguo xingzheng chufafa (1996), ZGFLNJ 1997, p. 236ff; deutsche Übersetzung bei Heuser (2003), note 7, p. 406ff.

Zhonghua renmin gongheguo xingzheng fuyifa (1999), ZGFLNJ 2000, p. 244ff; deutsche Übersetzung bei Heuser (2003), note 7, p. 318ff.

Zhonghua renmin gongheguo lifafa (2000), ZGFLNJ 2001, p. 257ff; deutsche Übersetzung bei Heuser (2003), note 7, p. 343ff.

Zhonghua renmin gongheguo xingzheng xukefa (2004), ZGFLNJ 2004, p. 240ff, deutsche Übersetzung bei Robert Heuser, Reduzierung und Normierung staatlicher Präventivintervention: Zum In-Kraft-Treten des chinesischen Verwaltungsgenehmigungsgesetzes, China Aktuell (CA) 2004, p. $288 \mathrm{ff}$. 2011-07/01/content_1897308.htm (zuletzt aufgerufen am 02.01.2012).

19

Vgl. Bu, note 8, p. 48f; Heuser (2003), note 7, p. 56. 
Erläuterungen finden sich jedoch in den Ansichten des Obersten Volksgerichts zum VPG. $^{20}$ Ein konkreter Verwaltungsakt ist diesen zufolge ,eine einseitige Handlung, die eine staatliche Verwaltungsbehörde [...] in Ausübung ihrer Verwaltungskompetenz gegenüber bestimmten Bürgern oder juristischen Personen [...] hinsichtlich eines konkreten Gegenstandes zur Regelung von Rechten und Pflichten (dieser Person) tätigt““ ${ }^{21}$ Demgegenüber sind abstrakte Verwaltungsakte Akte der Normsetzung zur Regelung einer unbestimmten bzw. unbestimmbaren Vielzahl zukünftiger Fälle. Hierunter fallen die abstrakt generellen und allgemeinverbindlichen Hoheitsakte der Verwaltung, insbesondere Verwaltungsrechtsbestimmungen (xingzheng fagui; im Ergebnis Rechtsverordnungen) und Verwaltungsvorschriften (xingzheng guizhang; im Ergebnis Satzungen). Letztere dienen einem Großteil der erlassenen konkreten Verwaltungsakte als Rechtsgrundlage. ${ }^{22}$

Neben dem einseitigen, finalimperativen Handeln in Form des Verwaltungsaktes, steht es der Verwaltung frei, „Verwaltungsverträge“ (xingzheng hetong, xingzheng qiyue, aber auch shuangfang xingzheng xingwei) zu schließen. Dieses kooperative Instrument findet erst im Fortgang der Reform- und Öffnungspolitik in der chinesischen Verwaltungsrechtslehre Beachtung ${ }^{23}$ und hat in der Praxis in den letzten zwei Jahrzehnten zunehmend an Bedeutung gewonnen. ${ }^{24}$ In westlichen Sprachen ist dieser Bereich noch nicht umfassend untersucht.

In der chinesischen Handlungslehre decken die Begriffe des konkreten und abstrakten Verwaltungsakts als Grund- bzw. Sammelkategorien alle Handlungsformen mit Regelungscharakter ab. In Abhängigkeit ihres Einzelfallbezuges werden sie entweder der einen oder der anderen Kategorie zugeordnet. ${ }^{26}$ Auch in China wird weiterhin anhand des Vorhandenseins einer Regelungswirkung zwischen Rechts- und Realakten unterschieden. ${ }^{27}$ Laut herrschender, aber bestrittener Meinung ${ }^{28}$, ist die Verwaltungsanleitung mangels Rege-

Zui gao renmin fayuan guanyu guanche zhixing ,Zhonghua renmin gongheguo xingzheng susongfa" ruogan wenti de yijian (shixing) (1991), Zhounghua renmin gongheguo zui gao renmin fayuan gongbao (ZGRFGB) [Amtsblatt des obersten Volksgerichtshofes] 1991, Nr. 3, p. 23ff; deutsche Übersetzung bei Heuser (2003), note 7, p. $295 \mathrm{ff}$. Übersetzung nach Heuser (2003), note 7, p. 57.

Vgl. Heuser (2003), note 7, p. 50ff.

23 Vgl. Jie Wu / Songnian Ying, Xingzhengfa [Verwaltungsrecht], ohne Ort 1986, p. 94f; Songnian Ying, Xingzhengfa jiaocheng [Lehrbuch der Verwaltungsrechtswissenschaft], Beijing 1989, p. 315ff; Shuyi Zhang, Xingzheng hetong [Der Verwaltungsvertrag], Beijing 1994. Vgl. Liu, note 7, p. 135. Vgl. Songnian Ying, note 23, p. 184ff.

27 Vgl. stellvertretend Yang Zhang / Lin Gao, Shilun xingzheng zhidao de jiuji [Über den Rechtsschutz gegen die Verwaltungsanleitung], Verwaltung und Recht (XZYF) 11/2007, p. 89. 
lungscharakter dem diffusen und sowohl im chinesischen als auch im deutschen Verwaltungsrecht ${ }^{29}$ dogmatisch nur unbefriedigend erfassten Bereich der Realakte zuzuordnen.

\section{Dogmatische Bestandsaufnahme}

Die chinesische Verwaltungsrechtswissenschaft scheint sich in einem Punkt weitgehend einig zu sein: Die Verwaltungsanleitung birgt großes Potential und ist dank ihrer Flexibilität und Effizienz für die wirtschaftliche und gesellschaftliche „Entwicklungsarbeit“ von nicht zu unterschätzendem Wert. Gleichzeitig gibt es aber eine Reihe schwerwiegender Probleme und ungelöster Fragen, die das Gesamtbild trüben. ${ }^{30}$ Bei den potentiellen Adressaten einer Verwaltungsanleitung kann sich dies bisweilen zu einer zwiespältigen Position ausweiten: „Anleitung erhoffen und fürchten“ („Xiwang zhidao you haipa zhidao") ${ }^{31}$. Zusammenfassend gesehen, kreisen die Probleme in der Hauptsache um zwei neuralgische Punkte: die Rechtmäßigkeit der Verwaltungsanleitung und der Rechtsschutz gegen sie. In beiden Punkten existieren einige Fragen und einige Probleme, für die bis heute keine zufriedenstellenden Antworten und Lösungen gefunden wurden. ${ }^{32}$

\section{Definitionsversuche}

Wie der Verwaltungsakt, so entbehrt auch die Verwaltungsanleitung einer Legaldefinition. Dies bietet einerseits Unsicherheit, andererseits aber auch Freiraum. Demzufolge enthält eine beträchtliche Zahl der Beiträge zu diesem Thema einen mehr oder weniger eigenen Definitionsversuch des Autors. Diese Definitionsversuche mögen auf den ersten Blick sprachlich und begrifflich nicht unerheblich variieren. Aus einer Zusammenschau ${ }^{33}$ von in etablierten verwaltungsrechtlichen Zeitschriften vertretenen Definitionen lassen sich jedoch einige Tatbestandsmerkmale destillieren, die beinahe alle Definitionsversuche gemeinsam haben. Der Veranschaulichung mag das folgende, vergleichsweise griffig formulierte Beispiel dienen:

Verwaltungsanleitungen sind „Anleitungen, Ratschläge, Empfehlungen und weitere Maßnahmen, die Verwaltungsbehörden im Rahmen ihrer Zuständigkeit aussprechen, um

29

30

31

32

33

Überblicksartig bei Maurer, note 3, § 15, Rn 1f.

Ausführlich Runsheng Guo / Gongde Song, Xingzheng zhidao [Die Verwaltungsanleitung], Beijing 1999, p. 81f.

Qingning Wang / Weidong Liu, Jiubu xingzheng zhidao dui fazhi xingzheng de pianli [Das Schließen der Kluft zwischen Verwaltungsanleitung und rechtsstaatlicher Verwaltung], XZYF 04/2004, p. 42.

Dazu sogleich in Kap. III. 3.

Vgl. für einen Überblick stellvertretend $Y u$ Cheng, Xingzheng zhidao xingzhi bianxi [Eine differenzierende Untersuchung über die Rechtsnatur der Verwaltungsanleitung], XZYF 12/2006, p. 75f; Zhuolan Cui / Pengyu Lu, Riben xingzheng zhidao zhidu jiqi falü kongzhi lilun [Das System der Verwaltungsanleitung in Japan und die Theorie ihrer rechtlichen Kontrolle], Xingzheng faxue yanjiu (XZFXYJ) [Verwaltungsrechtliche Forschung] 03/2001, p. 68f. 
bei den Angeleiteten durch ein Handeln oder Unterlassen ein Verwaltungsziel zu erreichen. Sie haben keine hoheitliche Zwangswirkung und lösen keine unmittelbaren Rechtsfolgen aus. “ 34

Will man die Verwaltungsanleitung also beschreiben, so müssen mindestens die hier genannten Tatbestandsmerkmale Beachtung finden. Es bleibt vorerst festzuhalten: Verwaltungsanleitungen werden von einem Verwaltungsorgan durchgeführt. Um ein bestimmtes Ziel zu erreichen, bedient sich dieses eines Bündels verschiedenartiger, jedoch nicht abschließend aufgezählter oder methodisch begrenzter Maßnahmen. Diese Maßnahmen und Ziele müssen im Rahmen der Aufgaben und Kompetenzen des jeweiligen Organs angesiedelt sein. Ob sie einer gesetzlichen Ermächtigungsgrundlage bedürfen, geht aus den Definitionen nicht eindeutig hervor. Ein Regelungscharakter oder eine Zwangswirkung werden verneint. Fast alle dieser Tatbestandsmerkmale sind in der chinesischen Verwaltungsrechtswissenschaft umstritten. Sowohl im Hinblick auf ihre Inhalte, als auch im Hinblick auf ihre Notwendigkeit.

\section{a) Maßnahmenkatalog}

Vergleichsweise geringe Schwierigkeiten bringt die Frage mit sich, welche konkreten Handlungen und Maßnahmen eine Verwaltungsanleitung eigentlich umfassen kann. Wie sich bereits aus dem Wortlaut (,und weitere Maßnahmen“) der meisten und auch aus dem der oben genannten Beispielsdefinition ergibt, existiert kein Katalog konkreter Maßnahmen. Die Verwaltungsanleitung ist in ihren Erscheinungsformen nicht begrenzt, sie ist kein geschlossenes System. Es handelt sich nicht um die Bezeichnung eines bestimmten, konkreten Verfahrens oder Vorgehens, sondern vielmehr um einen sprachlich unscharfen Sammelbegriff, unter den eine Vielzahl verschiedenartiger Handlungsvarianten subsumiert werden kann.

Nichtsdestotrotz hat sich, wie auch in Deutschland ${ }^{35}$, eine Typologie der informalen Verwaltungshandlungen herausgebildet. Die chinesische Rechtswissenschaft orientiert sich in der Regel an einer Einteilung aus der japanischen Verwaltungslehre der 1960er Jahre. ${ }^{36}$ Nach dieser Einteilung gibt es grundsätzlich drei Gruppen von Verwaltungsanleitungen:

Xia Yin / Guiping Zhu, Woguo xingzheng zhidao chengxu cunzai de wenti yu yingdui [Probleme und Gegenstrategien im Verfahren der Verwaltungsanleitung in China], XZYF 09/2008, p. 25. 
(quasi-)regelnde (guizhixing) ${ }^{37}$, justierende (tiaozhengxing) und begünstigende (shouyixing oder zhuchengxing) Verwaltungsanleitungen. Mischtypen sind jedoch denkbar. ${ }^{38}$

Unter begünstigenden Verwaltungsanleitungen versteht man unterstützende und fördernde Maßnahmen der Verwaltung gegenüber privaten Adressaten. Beispiele sind Maßnahmen der Beschäftigungsförderung oder der Information der Bevölkerung über gesetzliche Regelungen, Subventionen und technische Hilfsleistungen für Wirtschaftsunternehmen. ${ }^{39}$ Soweit ersichtlich, herrscht Einigkeit darüber, dass solche Maßnahmen die Rechte und Interessen der Adressaten nicht negativ beeinflussen. ${ }^{40}$ Darin liegt der zentrale Unterschied zu den quasi-regelnden und justierenden Verwaltungsanleitungen.

Bei den justierenden Verwaltungsanleitungen geht es um die Einflussnahme auf die Beziehungen zweier Adressaten untereinander oder auf die Beziehung eines Adressaten zum Verwaltungsträger selbst. Als Beispiel werden Ratschläge zur Beilegung privater Konflikte genannt, wie z.B. Streit zwischen den Parteien eines Hochhauses. Der Verwaltung wird hier, sicher nicht zuletzt aufgrund ihrer mit hoheitlichen Kompetenzen bewehrten, besonderen Stellung, großes Potential zur Konfliktlösung zugeschrieben. ${ }^{41}$

Durch quasi-regelnde Verwaltungsanleitungen soll rechtswidriges oder Gemeinwohl schädigendes Verhalten seitens der Adressaten unterbunden werden. Hierin liegen zumindest potentiell die größten Einfluss- und daher auch Missbrauchsmöglichkeiten der Verwaltung. Bereits das Konzept der quasi-regelnden Anleitung verspricht nur dann Erfolg, wenn eine gewisse Zwangswirkung von ihr ausgehen kann. Dementsprechend erfolgt sie meist in Form von Warnungen, Verhandlungen oder auch direkten Forderungen an die Adressaten zur Erreichung eines Handelns oder Unterlassens. Als Beispiele werden Abrissverfügungen, Einfluss auf Infrastrukturmaßnahmen, wie Straßenbau und die Verkleinerung großer Unternehmen genannt. ${ }^{42}$

Im Folgenden wird dieser Typ der Verwaltungsanleitung als „,quasi-regelnd“ bezeichnet, obwohl die wörtliche Übersetzung dies nicht stützt. Auf diese Weise wird deutlich, dass es sich auch bei diesem Typ nicht um Maßnahmen mit Regelungscharakter im rechtlichen Sinn handelt, sondern die regelnde Wirkung faktischer Art ist. Dazu sogleich.

Vgl. Yan Li, Lun jiang xingzheng zhidao naru woguo xingzheng susong shou'an fanwei - yi riben fayuan dui xingzheng zhidao de sifa shencha wei qishi he jiejian [Zur Aufnahme der Verwaltungsanleitung in den Katalog der Maßnahmen, gegen die Verwaltungsklage erhoben werden kann - Inspirationen und Erfahrungen des justiziellen Umgangs mit Verwaltungsanleitungen durch japanische Gerichte], XZFXYJ 03/2009, p. 63; Zheng Liu, Zhong mei xingzheng zhidao zhidu bijiao yanjiu [Eine vergleichende Untersuchung über die Systeme der Verwaltungsanleitung in China und den USA], XZYF 04/2006, p. 75.

Vgl. Cui / Lu, note 33, p. 70; Liu, note 38, p. 75; Yunchuan Mo, Xingzheng zhidao bijiao yanjiu [Eine vergleichende Studie der Verwaltungsanleitung], Bijiaofa yanjiu (BJFYJ) [Rechtsvergleichende Forschung] 12/2004, p. 81.

Vgl. Xiangfa Kong, Lun xingzheng zhidao de falü kongzhi [Zur gesetzlichen Kontrolle der Verwaltungsanleitung], XZYF 10/2006, p. 77.

$41 \mathrm{Vgl}$. Cui/Lu, note 33, p. 70; Kong, note 40, p. 77; Liu, note 38, p. 75.

42 Vgl. Cui/Lu, note 33, p. 70; Kong, note 40, p. 77; Liu, note 38, p. 75; Wang / Liu, note 31, p. 41. 
b) Verwaltungsorgan als Anleitender

Die Verwaltungsanleitung wird von jedem Verwaltungsorgan als Träger hoheitlicher Gewalt, gegenüber einem Adressaten vorgenommen, der seinerseits nicht mit hoheitlicher Gewalt ausgestattet ist. Soweit ersichtlich, ist der Gebrauch der Verwaltungsanleitung im Innenverhältnis, also zwischen zwei oder mehr Verwaltungsorganen, im Gegensatz zu Japan, nicht gebräuchlich. ${ }^{43}$

Nicht berechtigt, Verwaltungsanleitungen durchzuführen sind demnach alle Einrichtungen, die nicht der Verwaltung zugerechnet werden können, wie z.B. private oder politische Gruppen oder Wirtschaftsverbände. ${ }^{44}$

\section{c) Rechtsgrundlage}

Das Tatbestandsmerkmal des Zuständigkeitsrahmens berührt die Frage nach der Berechtigung zum Erlass von Verwaltungsanleitungen und damit die Frage nach der Notwendigkeit einer Rechtsgrundlage. Dies ist ein besonders problematisches und umstrittenes Feld.

Über das letzte Jahrzehnt hinweg scheint sich in der Frage nach der Notwendigkeit einer Rechtsgrundlage für Verwaltungsanleitungen eine herrschende Meinung herausgebildet zu haben. Zwar werden auch andere, tendenziell weitere oder engere Ansätze verfolgt, in der Regel wird jedoch verlangt, dass die Verwaltungsanleitung zumindest auf eine irgendwie geartete Ermächtigungsgrundlage gestützt werden kann, sei sie auch eine politische Richtlinie. ${ }^{45}$

Unter dieser Prämisse hat sich ein „dreistufiges“ System der Rechtsgrundlagen für die Verwaltungsanleitungen herausgebildet.

Die erste Stufe in diesem System und damit die unproblematischste Rechtsgrundlage, ist das Gesetz selbst. In vielen verschiedenen Gesetzen sind bisweilen Regelungen zu finden, in denen Verwaltungsträger pauschal zu Handlungen ermächtigt werden, die zu den Maßnahmen im Rahmen von Verwaltungsanleitungen gezählt werden können. So bestimmt beispielsweise Art. 8 Abs. 3 der chinesischen Verfassung ${ }^{46}$, dass der Staat die Entwicklung der Kollektivwirtschaft antreibt (guli) und anleitet (zhidao). Laut dem Produktqualitätsge-

Vgl. Runsheng Guo / Gongde Song, Xingzheng zhidao gainian jietan [Grenzuntersuchung des Konzepts der Verwaltungsanleitung], Shanxi daxue xuebao (SXDXXB) [Wissenschaftliches Journal der Universität Shanxi] 02/2000, p. 5.

45

46

Zhonghua renmin gongheguo xianfa (1982, i.d.F. von 2004), ZGFLNJ 2005, p. 251ff; i.d.F. von 1999 in deutscher Übersetzung bei Heuser (2003), note 7, p. 207ff; i.d.F. von 2004 in englischer Übersetzung abzurufen unter http://english.gov.cn/2005-08/05/content_20813.htm (zuletzt aufgerufen am 02.01.2012). 
setz von $1993^{47}$ treibt der Staat die Wirtschaft dazu an (guli), die Produktqualität durch Wissenschaft und Technik zu steigern. Die chinesische Verwaltungsrechtswissenschaft lässt diese allgemein gehaltenen, bisweilen generalklauselartig formulierten Befugnisse als gesetzliche Ermächtigungsgrundlagen ausreichen. ${ }^{48}$

Enthält ein Gesetz keine konkrete oder allgemeine Befugnisnorm für die Vornahme von Verwaltungsanleitungen, so kommt auf einer zweiten Stufe eine Ermächtigung durch eine ungeschriebene Rechtsgrundlage in Betracht. Könnten auf Grundlage einer gesetzlichen Regelung final-imperative Maßnahmen, wie ein regulärer Verwaltungsakt, ergriffen werden, so wird hierin auch eine ungeschriebene, implizite Ermächtigungsgrundlage für anleitende Maßnahmen gesehen. Diesem Gedanken liegt ein „Erst-Recht-Schluss“ zu Grunde: Wenn ein Gesetz zu stärker einschneidenden Maßnahmen, als einer Verwaltungsanleitung ermächtigt, so ermächtigt es erst recht zu der (per definitionem milderen) Verwaltungsanleitung selbst.

Finden sich indes weder ausdrückliche noch implizite Ermächtigungsgrundlagen im materiellen Verwaltungsrecht, so sollen diese auf einem dritten Wege herzuleiten sein. Verwaltungsorgane können durch eine allgemein formulierte Aufgabenzuweisung oder Zuständigkeitsvorschrift in ihrem Organisationsrecht ${ }^{49}$, durch allgemeine Rechtsprinzipien oder Grundsätze ${ }^{50}$ und auch durch politische Richtlinien gewissermaßen subsidiär ermächtigt werden. ${ }^{51}$ In der Literatur wird vermutet, dass die meisten Verwaltungsanleitungen auf derartige Grundlagen gestützt werden. ${ }^{52}$

Es schließt sich allerdings die Frage an, wann welche Art der Rechtsgrundlage genügen soll. Hier wird die oben beschriebene Typenbildung praktisch relevant. Die wohl herrschende Meinung fordert, an die Rechtsgrundlagen umso höhere Anforderungen zu stellen,

Zhonghua renmin gongheguo chanpin zhiliangfa (1993 i.d.F. 2000), ZGFLNJ 1994, p. 286ff; in deutscher Übersetzung bei Frank Münzel, Chinas Recht 22.2.93/1, abrufbar unter http://lehrstuhl.jura.uni-goettingen.de/chinarecht/930222.htm (zuletzt aufgerufen am 02.01.2012). Vgl. Jiwen Chen / Jiaojun Wu, Xingzheng zhidao de kunjing jiqi jiejue lujing tanxi [Analyse des Dilemmas der Verwaltungsanleitung und Wege zu dessen Lösung], XZYF 04/2005, p. 51.

Vgl. z.B. das „Gesetz über die Organisation der territorialen Volkskongresse und der territorialen Volksregierungen aller Ebenen“ Zhonghua renmin gongheguo difang ge ji renmin daibiao dahui he difang ge ji renmin zhengfu zuzhifa) (1979 i.d.F. 2004), ZGFLNJ 2005, p. 354ff; übersetzt bei Frank Münzel, Chinas Recht 1.7.79/1, abrufbar unter http://lehrstuhl.jura.uni-goettingen.de/ chinarecht/790701.htm (zuletzt aufgerufen am 02.01.2012).

50 Vgl. ausführlich dazu Franz-Josef Kemnade, Generalklauseln im chinesischen Zivilrecht, Köln 2010, p. 16ff, 38ff (bislang unveröffentlicht).

Vgl. Guo / Song, note 44, p. 5; Yunchuan Mo, Gonggong weiji guanli - Xingzheng zhidao cuoshi - Xingzheng yingji yuanze. Gonggong weiji guanli zhong de xingzheng zhidao cuoshi yinchu de xingzheng faxue sikao [Öffentliches Krisenmanagement - Maßnahmen der Verwaltungsanleitung - Grundsatz des Verwaltungsnotstandes. Verwaltungsrechtliche Erwägungen über Verwaltungsanleitungen im öffentlichen Krisenmanagement], XZFXYJ 04/2009, p. 74, 77; kritisch dazu Kong, note 40, p. 78. 
je schwerer der potentielle Einfluss auf die Rechte und Interessen der Adressaten wiegen kann. Konkret bedeutet dies, dass bei begünstigenden Verwaltungsanleitungen keine handlungsformspezifische Ermächtigungsnorm vorliegen muss, sondern der Verwaltungsträger im Rahmen seiner Kompetenzen und in Übereinstimmung mit seinem Organisationsrecht nach freiem Ermessen handeln darf. Ähnliches soll auch für die justierenden Verwaltungsanleitungen gelten. Dabei wird darauf verwiesen, dass dies notwendigerweise so sein müsse, da die für Verwaltungsanleitungen charakteristische Flexibilität ansonsten verloren ginge. Lediglich für die quasi-regelnden Maßnahmen wird eine speziellere, handlungsspezifische Rechtsgrundlage gefordert, um bei diesem, die Adressaten potentiell am meisten belastenden Typus Missbrauch effektiv vorzubeugen. ${ }^{53}$

Nur schwerlich kann man sich dem Eindruck erwehren, hier werde vom Ergebnis her gedacht und versucht, den Einsatz eines Instrumentes zu rechtfertigen, welches formal zwar nicht zu rechtfertigen ist, dessen Potential und Nutzen für den Verwaltungsträger aber beträchtlich sind. Bisweilen finden sich daher auch kritische Stimmen, welche die Verwaltungsanleitung für grundsätzlich unzulässig halten. Man argumentiert, dass die Verwaltung immer aufgrund eines formellen Gesetzes zu ihren Handlungen ermächtigt sein müsse (Totalvorbehalt des Gesetzes) und deswegen eine Handlungsform wie die Verwaltungsanleitung, für die dies bis heute nicht der Fall ist, mit den Grundsätzen der Rechtsstaatlichkeit, insbesondere mit der Forderung des § 1 VPG nach einem „Regieren auf der Grundlage der Gesetze" (yifa xingzheng), unvereinbar sei. ${ }^{54}$

\section{d) Zielsetzung}

Auch in der Frage nach den legitimen Zielen von Verwaltungsanleitungen herrscht eine gewisse Uneinigkeit. Im Ergebnis scheinen jedoch die chinesischen Behörden in dieser Hinsicht keinerlei Beschränkungen unterworfen zu sein. Zwar wird nach wie vor über eine Zweckbindung an das allgemeine Wohl des Staates und seiner Bevölkerung nachgedacht: Es soll vor allem indirekt, nämlich durch den positiven Einfluss auf die Entwicklung der sozialistischen Marktwirtschaft als Ganzes gesteigert werden. ${ }^{55}$ Aufgrund der nicht vorhandenen gesetzlichen Regelung der Verwaltungsanleitungen wird sich diese Forderung jedoch wohl nicht in der Rechtsrealität widerspiegeln.

Vgl. Guo / Song, note 30, p. 68; Kong, note 40, p. 77; für regelnde und justierende Verwaltungsanleitungen ähnlich Wang / Liu, note 31, p. 42.

Vgl. Hanguang Li / Xiaomei Zhou, Xingzheng zhidao xinlai baohu yuanze yanjiu [Eine Studie über den Grundsatz des Vertrauensschutzes bei der Verwaltungsanleitung], XZYF 04/2003, p. 17f; Wang / Liu, note 31, p. 41. 
e) Regelungscharakter

Bereits aus den verschiedenen Definitionsvarianten geht hervor, dass die Verwaltungsanleitungen keine rechtsverbindlichen Regelungen sind. Diesen gemäß sind sie weder konkret individuelle Regelung noch abstrakt generelle Norm. ${ }^{56}$ Sie haben daher keine unmittelbare „Rechtskraft“ (quanli) oder Bindungswirkung (yueshuli) ${ }^{57}$, in deutscher Terminologie Regelungswirkung. Ihre Befolgung ist daher nicht rechtlich zwingend, ihre Durchsetzung nicht im Wege der Verwaltungsvollstreckung erzwingbar.

Der zentrale Streit um die Verwaltungsanleitung entzündet sich genau an diesem Punkt: Geht von der Verwaltungsanleitung trotz nicht vorhandener Regelungsqualität dennoch eine faktische Zwangswirkung aus und wie ist dies ggf. zu bewerten?

Das Regelungsmerkmal dient auch im chinesischen Verwaltungsrecht gerade der Abgrenzung zu den unverbindlichen, nicht zwingenden Handlungsformen. ${ }^{58}$ Bei den Maßnahmen der Verwaltungsanleitung wird dies zum Problem: Hier entstehen regelmäßig Situationen, in denen mangels Regelungscharakter eindeutig kein Verwaltungsakt vorliegt, aber dennoch ein bestimmtes Verhalten erwünscht und beabsichtigt wird. Dieser Widerspruch wird auch in der chinesischen Literatur wahrgenommen und diskutiert. Verbreitet stellt man sich dort auf den Standpunkt, die Befolgung der Verwaltungsanleitungen geschehe freiwillig. ${ }^{59}$ Diese Darstellung entpuppt sich jedoch dann als bloßes Scheinargument, wenn die Wahl an sich zwar möglich ist, aber im Falle einer bestimmten Entscheidung negative Konsequenzen zu erwarten sind. Wird durch eine Verwaltungsanleitung Druck auf die Adressaten ausgeübt, so dass diese die Wahl zwischen Befolgen der Anweisungen oder künftigen Repressalien, Nachteilen oder wirtschaftlichen Verlusten haben, so kann man nicht mehr von einer freiwilligen, unbeeinflussten Befolgung sprechen. Derartiges Verhalten der Behörden ist nach der aktuellen Lage des chinesischen Rechts de facto möglich. ${ }^{60}$ Verwaltungsträger und Bürger stehen sich trotz „freiwilliger Befolgung“ im Ergebnis doch nicht als Gleiche gegenüber. Der tatsächliche Charakter von Verwaltungsanleitungen ver-

56

57

58

59

60

Vgl. oben Kap. III. 1.

Vgl. statt vieler $L i$, note 38, p. 60; Sibin Peng, Xingzheng zhidao kesuxing bijiao qianxi [Eine vergleichende oberflächliche Studie über die Klagemöglichkeit gegen die Verwaltungsanleitung] XZYF 12/2002, p. 22.

Vgl. Songnian Ying, Xingzheng faxue xin lun [Neue Überlegungen zur Verwaltungsrechtslehre], Beijing 1999, p. 194ff.

Vgl. das Definitionsbeispiel aus Kap. III. 1; sowie stellvertretend Ziyin Gao, Qianyi xingzheng zhidao yu falü jiuji [Eine oberflächliche Analyse der Verwaltungsanleitung und gesetzlichen Rechtsschutzes], XZYF 07/2002, p. 28.

Statt vieler Xiaohong Duan, Xingzheng zhidao jiuji zhidu de biyao tanjiu [Eine Studie über die Notwendigkeit eines Rechtsschutzsystems gegen die Verwaltungsanleitung], XZYF 01/2003, p. 12f (mit Beispielen); Yunchuan Mo, Xingzheng zhidao jiuji zhidu yanjiu [Eine Studie des Rechtsschutzsystems gegen die Verwaltungsanleitung.], Faxuejia (FXJ) [Der Jurist] 05/2004, p. 131. 
mag so nicht abgebildet zu werden. ${ }^{61}$ Aufgrund des vermittelten Drucks seien diese eher als „Regulativbeziehung“ zu beschreiben, „die auf spezifischen außerrechtlichen Zwangsmethoden basiert". 62

Konsequent erscheint außerdem der Ansatz, Verwaltungsmaßnahmen, die einen faktischen Zwang beinhalten, bereits auf Tatbestandsebene nicht als Verwaltungsanleitung anzusehen, sondern inklusive aller einhergehender prozessualer Folgen als Verwaltungszwang bzw. -befehl, jedenfalls aber als Verwaltungsakt, einzustufen. Verwaltungsanleitungen wären demzufolge nur dann anzunehmen, wenn eine Maßnahme tatsächlich keine Zwangswirkungen entfaltet. ${ }^{63}$ Diese Ansicht hat sich jedoch bis jetzt nicht durchgesetzt.

\section{Rechtsnatur der Verwaltungsanleitung}

Die Diskussionen um das Tatbestandsmerkmal der Regelung legen bereits nahe, dass auch die Rechtsnatur von Verwaltungsanleitungen umstritten ist. In Betracht gezogen wurden und werden diesbezüglich zunächst zwei Varianten. Es könnte sich bei den Maßnahmen um Verwaltungsakte oder Realakte handeln.

Das chinesische Verständnis eines konkreten Verwaltungsakts, wie es oben gezeigt wurde, scheint auf den ersten Blick ungeeignet die Verwaltungsanleitung abzubilden. Dennoch werben Literaturstimmen für ein weites Verständnis des Regelungsmerkmals und damit für die Zuordnung zu den Verwaltungsakten. ${ }^{64}$

Dem steht die Ansicht gegenüber, dass die Handlungsformen der Verwaltungsanleitung als Realakte zu klassifizieren sind. ${ }^{65}$ Hier wird das Regelungsmerkmal eng ausgelegt und das informale Handeln konsequenter Weise den Realakten beigeordnet. Dies entspricht der wohl herrschenden Meinung und den Ansichten des Obersten Volksgerichtes. Dies legt dessen Auslegung des $§ 1$ Nr. 4 VPG seinem Wortsinne nach in den „Interpretationen von einigen Fragen zur Durchführung des Verwaltungsprozessgesetzes der VR China“66 auch nahe. Dort wird die Verwaltungsanleitung als ,nicht zwingende Handlung“ (bu juyou qiangzhili de [...] xingwei) bezeichnet. Auch das Gericht scheint davon auszugehen, dass eine zwangsweise Durchsetzung auf legalem Wege, nicht möglich ist und es sich nicht um eine rechtlich verbindliche Regelung handelt.

Vgl. Duan, note 60, p. 13; Ning Fang / Jin Zhu, Xingzheng zhidao kesuxing tanxi [Analyse der Klagemöglichkeit gegen die Verwaltungsanleitung], XZYF 04/2003, p. 15.

Walkling, note 1, p. 66.

Vgl. Fang / Zhu, note 61, p. 15; Huiping Zhang, Xingzheng zhidao jiben wenti tanlüe [Eine kurze Untersuchung über die grundlegenden Probleme der Verwaltungsanweisung], XZYF 01/2004, p. 68.

Vgl. nur Cheng, note 33, p. 77f; Guo / Song, note 44, p.7f.

Vgl. Duan, note 60, p. 12; Gao, note 59, p. 28; Zhang / Gao, note 27, p. 89.

66

Zui gao renmin fayuan guanyu zhixing ,Zhonghua renmin gongheguo xingzheng susongfa“ ruogan wenti de jieshi (1999), ZGRFGB 2000, S. 87ff; deutsche Übersetzung bei Heuser (2003), note 7 , p. $269 \mathrm{ff}$. 


\section{Probleme und Verbesserungsstrategien}

a) Rechtmäßigkeit

Aus der Analyse der Tatbestandsmerkmale wird ersichtlich, dass es sich bei der Verwaltungsanleitung um eine Vorgehensweise handelt, die Behörden in die Lage versetzt ihre Ziele nach freiem Ermessen festzusetzen und dann vielgestaltig zu verfolgen, ohne aber an die mit der Verwaltungsaktsqualität einhergehenden Formalien oder Überprüfbarkeit gebunden zu sein. Das Missbrauchspotential ist evident, die Transparenz der Maßnahmen nicht gewährleistet. Dies wird auch in China selbst, mitunter auch erstaunlich scharf, verurteilt.

Chinesischen Verwaltungsbeamten wird vorgeworfen, die traditionellen Denkstrukturen der Vergangenheit bezüglich der Stellung und Macht der Verwaltung nicht überwunden zu haben und die Verwaltungsanleitung mit Beeinflussungsmaßnahmen aller Art zu flankieren. So werde sie im Ergebnis wie ein Verwaltungsbefehl gebraucht. ${ }^{67}$ Das im kollektiven chinesischen Bewusstsein nach wie vor tief verwurzelte Konzept einer autoritären, lenkenden Verwaltung ${ }^{68}$ ermögliche und begünstige missbräuchliches Verhalten der Behörden. Werde eine Anleitung nicht freiwillig befolgt, so würden die Angeleiteten durch wirtschaftlichen und finanziellen Druck oder andere Repressalien zur Befolgung bewegt. Damit komme der Verwaltungsanleitung faktisch eine Zwangswirkung zu, die Freiwilligkeit werde den Angeleiteten genommen. ${ }^{69}$ Ganz grundsätzlich ist festzuhalten, dass den chinesischen Verwaltungsbeamten misstraut wird, mindestens aber ihre Motive als potentiell zweifelhaft wahrgenommen werden. ${ }^{70}$ Zusätzlich schätzt man die fachliche Qualifikation der Beamten als eher niedrig, ihre Anfälligkeit für Korruption und Machtmissbrauch dagegen hoch ein. ${ }^{71}$

Der Vorwurf, die Verwaltungsanleitung sei im Ganzen intransparent, wird unter anderem zu Recht damit begründet, dass es ohne Formvorschriften für die Adressaten in vielen Fällen nur schwer zu beurteilen sein wird, welcher Handlungsform sich die Verwaltung gerade bedient. Ob man Adressat eines Verwaltungsaktes oder einer Verwaltungsanleitung ist, mag bisweilen schwer auszumachen sein. Selbst ,innerhalb“ der Verwaltungsanleitungen schaffen die schwammigen und zum Teil nicht klar abgrenzbaren Bezeichnungen, wie z.B. Rat und Empfehlungen, Anleitung und Führung noch weitere Unklarheiten.

Vgl. Gao, note 59, p. 29; Mo, note 60, p. 131; Yin / Zhu, note 34, p. 25f; Zhang / Gao, note 27, p. 90 .

Vgl. zu den historischen Aspekten Heuser (2002), note 7, p. 56ff, 64ff.

Vgl. nur Chen / Wu, note 48, p. 51; Duan, note 60, p. 12f; Liu, note 38, p. 77; Zhang / Gao, note 27, p. 90.

70 Vgl. z.B. Mo, note 60, p. 131; Min Zhou, Guanyu gaijin xingzheng zhidao de sikao [Überlegungen zur Verbesserung der Verwaltungsanleitung], XZYF 06/2003, p. 69.

71 Vgl. Chen / Wu, note 48, p. 51; Duan, note 60, p. 13; Kong, note 40, p. 78; Liu, note 38, p. 75, 78 . 
Die Verwaltungsrechtswissenschaft bringt jedoch auch zahlreiche Vorschläge zur Verbesserung der aktuellen Situation vor. Eine der häufigsten Forderungen der Wissenschaft ist die, nach der gesetzlichen Regelung des Instituts der Verwaltungsanleitung, also ihrer Verrechtlichung. Diese soll allgemein und verbindlich sein und in der Regel wird vorgeschlagen, sie in das noch zu erlassende allgemeine Verwaltungsverfahrensgesetz zu integrieren. ${ }^{72}$ Damit wäre die Verwaltungsanleitung eindeutig dem Vorrang des Gesetzes unterworfen. So lange dies nicht geschehen ist, soll hilfsweise auf allgemeine Rechtsgrundsätze, wie den Vertrauensschutz und den Grundsatz der Freiwilligkeit, zurückgegriffen werden. ${ }^{73}$ Man erhofft sich durch die Verrechtlichung der Verwaltungsanleitung dem Interesse der Verwaltung an flexiblen Handlungsformen und dem Interesse der Bürger an möglichst lückenlosem Rechtsschutz Rechnung tragen zu können. Dabei wird jedoch nicht verkannt, dass sich gerade in Bezug auf die Verwaltungsanleitung ein Spannungsfeld aufbaut und allseitige Zufriedenheit schwer herzustellen sein dürfte. Transparenz der Inhalte und Bindung der Behörden an ein Verfahren auf der einen Seite, steht das Interesse der Verwaltung an einem flexiblen Steuerungsinstrument mit möglichst weitem Ermessensfreiraum auf der anderen Seite gegenüber. Die Vorteile der Verwaltungsanleitungen liegen ja gerade in ihrer Flexibilität, Informalität und Wirksamkeit. ${ }^{74}$

\section{b) Rechtsschutz}

Das zentrale und wohl am häufigsten diskutierte Problem im Umfeld der Verwaltungsanleitung ist die Frage des Rechtsschutzes. ${ }^{75}$ Die Gesetzeslage scheint jedoch eindeutig und die Diskussion darüber, ob die Fälle der Verwaltungsanleitung von den Gerichten überprüft werden können oder müssen, ist bis heute rein akademischer Natur. ${ }^{76}$

Der Grund dafür ist im geltenden Verwaltungsprozessrecht zu suchen. Das VPG und die Ansichten und Interpretationen des Obersten Volksgerichtes regeln im Gegensatz zur deutschen VwGO den Zugang zu den Verwaltungsgerichten nicht durch ein Generalklauselprinzip, sondern durch das Enumerationsprinzip. Das Regel-Ausnahme-Verhältnis wird umgekehrt und der Rechtsweg ist nur dann eröffnet, wenn das VPG oder die Ansichten dazu dies positiv bestimmen. Dies ist in den Katalogen der $\S \$ 11,12$ VPG geschehen. Nur gegen (konkrete) Verwaltungsakte, die in $\S 11$ VPG ausdrücklich als gerichtlich überprüfbar aufzählt werden und in $\S 12$ VPG nicht ausdrücklich als von der Überprüfbarkeit aus-

Vgl. z.B. Chen / Wu, note 48, p. 52; Yunchuan Mo, Lun xingzheng zhidao de lifa yueshu [Die Bindung der Verwaltungsanleitung an das Gesetz], Zhongguo faxue (ZGFX) [Recht in China] 02/2004, p. 52ff; Peng, note 57, p. 23.

Vgl. statt vieler $M o$, note 72, p. 48.

74

Vgl. statt vieler Mo, note 72, p. 46; Yin / Zhu, note 34, p. $25 \mathrm{f}$.

75 Vgl. stellvertretend für die große Mehrheit der Veröffentlichungen Chen / $W u$, note 48, p. 51; Kong, note 40, p. 78f; Mo, note 60, p. 131, $133 \mathrm{ff}$.

76

Vgl. $L i$, note 38 , p. 60 . 
genommen werden, ist der Verwaltungsrechtsweg eröffnet. ${ }^{77}$ Die Verwaltungsanleitung ist aber nach Ansicht des Obersten Volksgerichts kein Verwaltungsakt. Soweit darüber jedoch Unklarheit bestand, räumte das Gericht diese aus, als es in $\S 1 \mathrm{Nr} .4$ seiner Interpretationen zum VPG aus dem Jahr $1999^{78}$,nicht zwingende Akte der Verwaltungsanleitung“ der verwaltungsprozessualen Kontrolle ausdrücklich entzog. Rechtsschutz vor den Verwaltungsgerichten ist gegen Maßnahmen der Verwaltungsanleitung daher nicht zu erlangen.

Zahlreiche Literaturstimmen suchen dennoch nach Möglichkeiten die Verwaltungsanleitung gerichtlicher Überprüfung zugänglich zu machen und diesen „,blinden Fleck des Rechtsschutzes“" ${ }^{79}$ zu beseitigen. ${ }^{80}$ Im Detail unterscheiden diese sich zwar, grundsätzlich betrachtet lassen sich aber Gemeinsamkeiten feststellen.

Man verweist in der Hauptsache auf die Anwendung allgemeiner Rechtsgrundsätze. Der Grundgedanke des Rechtsschutzes gegen die Verwaltungsanleitung auf der Grundlage von allgemeinen Rechtsgrundsätzen ist die Annahme, dass Rechtsbefugnisse und Rechtsschutz untrennbar miteinander verbunden sind. Wer ein Recht ausübt, muss für eventuell entstehende Folgen haften. ${ }^{81}$ Beansprucht also ein Verwaltungsorgan sein Recht, eine Verwaltungsanleitung durchzuführen, so soll es die Verantwortung für die Folgen und das Risiko tragen, ggf. Ansprüchen der Adressaten ausgesetzt zu sein. Es gilt der Grundsatz: Jeder Akteur haftet für eigenes Verschulden. Schuldhaft sollen die Adressaten dann gehandelt haben, wenn sie einer Ermahnung in Gestalt einer Verwaltungsanleitung, ein rechtswidriges Verhalten einzustellen, nicht Folge leisten oder wenn sie einer offensichtlich rechtswidrigen Verwaltungsanleitung trotz positiven Wissens darum entsprechen. Letzteres dürfte in der Regel Fälle von Verfolgungen eigener, ggf. auf legalem Wege nicht erreichbarer Ziele betreffen. Die Verwaltungsträger handeln ihrerseits schuldhaft, wenn sie wissentlich rechtswidrige oder unlautere Verwaltungsanweisungen erlassen, zurechenbar gesetztes schutzwürdiges Vertrauen enttäuschen oder faktischen Zwang auf die Adressaten ausüben. Entsteht ein Schaden im Zusammenhang einer regulären, ,normal“ verlaufenden Verwaltungsanleitung aufgrund der freiwilligen Befolgung seitens der Adressaten, so sollen sie selbst haften.

Besonders betont wird dabei der Schutz des Vertrauens des angeleiteten Adressaten in die Verwaltung. ${ }^{82}$ Schutzwürdiges Vertrauen bzw. ,berechtigte Erwartungen“ (hefa yuqi) können entstehen, wenn ein Verwaltungsträger im Rahmen seines Umgangs mit Privaten

77

78

79

80

81

82

Vgl. zur Systematik des chinesischen Verwaltungsprozessrechts Liu, note 7, p. 63ff.

Vgl. oben note 66, deutsche Übersetzung bei Heuser (2003), note 7, p. $269 \mathrm{ff}$.

Zhou, note 70, p. 69.

Vgl. die deutliche Kritik bei $\mathrm{Li} /$ Zhou, note 54, p. 17.

Vgl. z.B. Xianggang Li, Lun woguo xingzheng zhidao zhidu de gaishan [Zur Perfektionierung des chinesischen Systems der Verwaltungsanleitung], XZYF 09/2006, p. 78; Sun, note 55, p. 39; Peng Zhang, Xingzheng zhidao de zerenhua mantan [Eine informelle Diskussion über die Entwicklung der Haftung für Verwaltungsanleitungen.], XZYF 03/2001, p. 47.

Vgl. Li / Zhou, note 54, p. 17ff; Liu, note 38, p. 78; Yin / Zhu, note 34, p. 26. 
zurechenbar eine Vertrauensposition in Anspruch nimmt. Leitet also eine Behörde einen Privaten an, so könnte allein durch die dieser Anleitung innewohnende Autorität für den Angeleiteten schutzwürdiges Vertrauen dahingehend entstehen, dass er sich auf Angaben der Verwaltung verlassen und entsprechend disponieren kann. Darüber hinaus soll die Anleitung einer Behörde den Angeleiteten berechtigterweise erwarten lassen können, dass für die Behörde das eigene Wort bindend ist. Es wird vorgeschlagen in solchen Fällen zunächst, soweit möglich, den Bestandsschutz der Verwaltungsanleitung sicherzustellen. Sollte dies nicht möglich sein, so soll eine Entschädigung in Geld erfolgen. ${ }^{83}$

Tatsächlich scheint es in der Gerichtspraxis gewisse Aufweichungstendenzen zu geben. Der Verwaltungsrechtler Mo Yunchuan bemerkte bereits vor einigen Jahren einen erfreulichen Trend. Laut $M o$ haben einige (erstinstanzliche) Unterstufengerichte damit begonnen, Maßnahmen der Verwaltungsanleitung trotz der oben beschriebenen verwaltungsprozessualen Regelung zu überprüfen. ${ }^{84}$ Dabei handelt es sich jedoch, soweit ersichtlich, um vereinzelte Fälle, die als Ausnahmen zu werten sein dürften. Über den weiteren Verlauf dieser Verhandlungen und Entscheidungen über eventuell eingelegte Rechtsmittel wird nicht berichtet.

Als grundsätzlich überraschend ist anzumerken, dass der Rechtsschutz von Dritten, also nicht Anleitenden oder Adressaten, wenig bis gar nicht problematisiert wird. Eine entsprechende Problematik liegt meines Erachtens jedoch auf der Hand; eine eingehende Analyse bleibt daher ein Desiderat. ${ }^{85}$

\section{IV. Überblick über die Einsatzgebiete}

Mit der Abkehr von der planwirtschaftlich organisierten Wirtschaft und der stufenweisen Öffnung Chinas für markt- und privatwirtschaftliche Mechanismen und Konzepte zog sich der Staat ein Stück weit aus diesem Gebiet zurück. ${ }^{86}$ Das Bedürfnis nach Regulation und Steuerung ging aber keineswegs verloren. Die Verwaltungsanleitung wird als ein effektives Mittel gesehen, diesem Bedürfnis nachzukommen, gleichzeitig aber die Eigenverantwort-

Vgl. $\mathrm{Li}$ / Zhou, note 54, p. 19; Lingyun Yu, Xingzheng zhidao zhizhong de hefa yuqi - dui Quanzhou gongshangju shijian jingyan de kaocha yu sikao [Berechtigte Erwartungen bei der Verwaltungsanleitung - Untersuchungen und Reflexionen über die Praxis und Erfahrungen der Handelskammer in Quanzhou], FXJ 05/2007, p. 51f.

Vgl. Mo, note 60, p. 132, 135 .

Vgl. lediglich kurz bei Wenyu Song, Xingzheng zhidao chengdan falü zeren de biyaoxing fenxi [Eine Analyse der Notwendigkeit gesetzlicher Haftung für die Verwaltungsanleitung], Faxue zazhi (FXZZ) [Journal der Rechtswissenschaft] 05/2002, p. 26.

Vgl. überblicksartig Carsten Herrmann-Pillrath, Wirtschaftsordnung, in: Staiger / Friedrich / Schütte (eds.), note 7, p. 852ff, 934 mit weiteren Nachweisen; Markus Taube, Wirtschaftliche Entwicklung und struktureller Wandel seit 1949, in: Doris Fischer / Michael Lackner (eds.), Länderbericht China Geschichte - Politik Wirtschaft - Gesellschaft, 3. Aufl., Bonn 2007, p. 248ff mit weiteren Nachweisen. 
lichkeit der privaten Wirtschaftsakteure zu erhalten und zu betonen. ${ }^{87}$ Vor allem soll der schnellen Entwicklung hin zu einem technisch wie organisatorisch immer komplexer vernetzten Wirtschafts- und Gesellschaftssystem Rechnung getragen werden. Die Gebiete, auf denen die Verwaltungsanleitung in China Einsatz findet, können hier nur sehr schematisch skizziert werden. Es finden sich keine Quellen, die schlussfolgern lassen, der Einsatz von Verwaltungsanleitungen beschränke sich auf bestimmte, klar abgrenzbare Bereiche. Vielmehr muss angenommen werden, dass die Behörden sie flexibel und flächendeckend einsetzen können.

Es verwundert nicht, dass das Gebiet der Wirtschaftssteuerung das zentrale Einsatzfeld der Verwaltungsanleitung darstellt. ${ }^{88}$ Darunter fällt auch die Landwirtschaft. Neben diesen klassischen Einsatzgebieten der Verwaltungsanleitung ist in den letzten Jahren deren Potential und Anwendung im Bereich des öffentlichen Krisenmanagements in den Mittelpunkt des Interesses gerückt. Obwohl aus dem westlichen Ausland teils massive Kritik an chinesischem Krisenmanagement vorgetragen wurde ${ }^{89}$, ist man überzeugt, mit der Verwaltungsanleitung ein Mittel zur Hand zu haben, welches insbesondere in Situationen, in denen geplantes und förmliches Handeln aufgrund großen Zeitdrucks nicht praktikabel wäre, ihr volles Potential entfalten kann und wird. ${ }^{90}$

In jüngster Vergangenheit wurde der Einsatz von Maßnahmen der Verwaltungsanleitung sogar für die Eindämmung der Folgen der internationalen Finanz- und Wirtschaftskrise diskutiert. Es wurde gefordert, sowohl schadensbegrenzende Maßnahmen als auch Finanzmarktregulierungen nicht nur auf dem ,klassischen“ final-imperativen Weg, sondern zumindest auch im Wege kooperativer Steuerungsverwaltung durchzusetzen. Auf diese Weise könne man den Anforderungen dieses komplexen Sektors besser gerecht werden. ${ }^{91}$

\section{Bewertung}

Die im Gegensatz zu anderen ostasiatischen Staaten bis heute nicht erfolgte gesetzliche Regelung der Verwaltungsanleitung fördert eine lebhafte Diskussion. Diese scheint ange-

87

88

89

90

Vgl. Wojie Meng, Yingji guanli zhong de xingzheng zhidao wenti lüelun [Abhandlung über Fragen der Verwaltungsanleitung im Krisenmanagement.] XZYF Bd. 11/2009, p. 44; Qing Nie, Lun gonggong weiji guanli zhong de xingzheng zhidao [Zur Verwaltungsanleitung im öffentlichen Krisenmanagement.], XZYF Bd. 01/2007, p. 17.

91

Vgl. Yunchuan Mo, Ying jiji fahui xingzheng zhidao cuoshi zai yingdui guoji jinrong weiji jizhong zhong de zuoyong - jianlun tongguo xingzheng guanli fangshi chuangxin tisheng zhengfu de weiji ganyu nengli [Der Nutzen von Maßnahmen der Verwaltungsanleitung sollte im Rahmen der Bekämpfung der internationalen Finanzkrise positiv zur Geltung kommen - über die Innovationen und Verbesserungen der staatlichen Krisenintervention durch die Art der Verwaltung], XZFXYJ 04/2009, p. 77. 
sichts der offenkundigen Ambivalenz dieser Handlungsform so angebracht wie notwendig. Man ist sich der rechtlichen und tatsächlichen Gefahren und Widersprüche durchaus bewusst, erkennt aber gleichzeitig den enormen Nutzen und das Potential dieses Konzepts für die Lenkung des eigenen Staates.

Legt man den Maßstab des Rechtsstaates westlicher Prägung an, so sind Handlungen des Staates, die ohne Rechtsgrundlage erfolgen und den Instanzen des Rechtsschutzes entzogen sind, zweifellos hoch problematisch. Insoweit berührt die Betrachtung der Problematiken um die Verwaltungsanleitung die (verfassungs-)rechtlichen Grundfesten und Grundentscheidungen der Volksrepublik. Das Spannungsfeld und der Widerspruch zwischen dem Selbstanspruch, den Staat „auf der Grundlage der Gesetze“ zu verwalten und dem Primat der Politik über das Recht werden hier deutlich spürbar. Der ,genuin chinesische Umgang “92 mit der Verwaltungsanleitung verdient schon deswegen Aufmerksamkeit. In Anbetracht der internationalen Verbreitung informaler Verwaltungspraktiken erscheint es jedoch zu einfach und stereotyp, die Verwaltungsanleitung als „typisch chinesisch“ abzutun und in die lange Reihe problematischer Phänomene in China einzuordnen. Eine derartige Aussage ließe sich, wenn überhaupt, nur in einer kultur-soziologischen Studie gewinnen. Für die innerchinesische Perspektive bleibt festzuhalten: Die chinesischen Juristen werten ihre Vergangenheit und die traditionell sehr starke Position der Verwaltung zwar als einen begünstigenden Faktor für den Erfolg, aber auch für den Missbrauch der Verwaltungsanleitung. ${ }^{93}$ Ingesamt lässt sich der Erfolg der Verwaltungsanleitung aber schlüssiger auf ihren praktisch einstimmig hervorgehobenen wirtschaftlichen Nutzen zurückführen. Eine solche Betrachtung würde dem Institut den Nimbus des Esoterisch-Asiatischen nehmen und deutlich machen, dass auch informale Handlungsformen „Zweckschöpfungen des Verwaltungsrechts ${ }^{\text {" }}{ }^{94}$ sind.

Der Erlass des allgemeinen VwVfG der VR China birgt die Möglichkeit, die Verwaltungsanleitung zu regeln. Ob der chinesische Gesetzgeber diese nutzen wird, ist nicht mit Sicherheit vorauszusagen, würde aber in Anbetracht der zwar meist höflichen, aber doch unüberhörbar klaren Kritik aus den Reihen der juristischen Fachwelt des Landes nicht überraschen. Es wäre eine gute Möglichkeit zu zeigen, dass sich die Waage im Konflikt zwischen Rechtstaatsbestrebungen und Gewalteneinheit ein wenig mehr zur Rechtsstaatlichkeit neigt.

Vgl. Kap. III. 3. a).

94

Franz-Josef Peine, Allgemeines Verwaltungsrecht, 9. Aufl., Heidelberg 2008, Rn. 308. 